\title{
NON LINEAR SCHRÖDINGER EQUATIONS - APPROXIMATE CONTROLLABILITY IN NONCYLINDRICAL DOMAINS
}

\section{Ricardo F. Apolaya ${ }^{1}$}

ABSTRACT.- We consider $\Omega \subset \mathbb{R}^{n}$ an open bounded set and $\varphi$ be a complex and globally Lipschitz function.

In this work we investigate approximate controllability for the non linear Schrödinger Equation in Noncylindrical Domains:

$$
\mid \begin{aligned}
& u^{\prime}-i \Delta u+\varphi(u)=v \cdot \chi_{\omega} \text { in } \hat{Q} \\
& u=0 \text { in } \hat{\Sigma} \\
& u(x, 0)=u_{0}(x) \text { in } \Omega_{0},
\end{aligned} \quad\left(i^{2}=-1\right)
$$

$v=v(x, t)$ represents the control function and $\chi_{\omega}$ is the characteristic function of $\omega$. The noncylindrical domain is defined by a family of orthogonal matrix $K(t)$. By' we denote the derivative with respect to the time variable. We say that system $(*)$ is approximately controllable in $L^{2}(\Omega)$ at time $T>0$ if the following holds: "For every $u_{0} \in L^{2}(\Omega)$ the set reachable states at time $T>0, E(T)=\left\{u(x, T)\right.$, u is the solution of $\left.\left({ }^{*}\right)\right\}$ is dense in $L^{2}\left(\Omega_{T}\right)^{\prime \prime}$.

KEYWORDS.- Schrödinger Equations. Non linear Approximate Controllability. Noncylindrical Domains.

\section{CONTROLABILIDAD APROXIMADA DE ECUACIONES DE SCHÖDINGER NO' LINEALES EN DOMINIOS NO CILÍNDRICOS}

RESUMEN.- Consideremos $\Omega \subset \mathbb{R}^{n}$ un conjunto abierto acotado y sea $\varphi$ una función compleja globalmente Lipschitziana. En este trabajo investigamos la controlabilidad aproximada para la ecuación de Schrodinger no lineal en dominios cilindros $(*)$, donde $v=v(x, t)$ representa la función de control y $\chi_{\omega}$ es la función característica de $\omega$. El dominio no cilíndrico es definido por una familia de matrices ortogonales $K(t)$. Por 'denotamos la derivada con respecto a la variable tiempo.

Decimos que el sistema $(*)$ es controlable aproximadamente en $L^{2}(\Omega)$ en el tiempo $T>0$ si se cumple lo siguiente: "Para caa'a $u_{0} \in L^{2}(\Omega)$ el conjunto observado en el tiempo $T>0, E(T)=\{u(x, T)$, u es la solución de (*) $\}$ en denso en $L^{2}\left(\Omega_{T}\right)$ ".

PALABRAS CLAVE.- Ecuación de Schrödinger no lineal. Controlabilidad Aproximada. Dominios no cilíndricos.

\footnotetext{
'Instituto de Matemática UFF-Niteroi-RJ Brasil e-mail: ricardof16@yahoo.com.br
} 


\section{INTRODUCTION}

The aim of this article is to show some results that objectify the obtaining of the exact boundary controllability for Schrödinger equation in a noncylindrical domain.

Let $\Omega$ be a bounded open domain in $\mathbb{R}^{n}$ having a boundary $\Gamma$ of class $C^{2}$. It is also supposed that $\Omega$ contains the origin of $\mathbb{R}^{n}$. We denote by $K(t)$ an orthogonal matrix defined on $[0, \infty[$. For each $t \in[0, T], 0<T<\infty$ we define the sets

$$
\begin{gathered}
\Omega_{t}=\left\{x \in \mathbb{R}^{n} ; x=K(t) y, y \in \Omega\right\}, \Gamma_{t}=\partial \Omega_{t}, \\
\hat{Q}=\bigcup_{0<t<T} \Omega_{t} \times\{t\}(\hat{Q}=\text { noncylindrical domain }), \hat{\Sigma}=\bigcup_{0<t<T} \Gamma_{t} \times\{t\} .
\end{gathered}
$$

This paper is devoted to the existence, uniqueness and approximate controllability of nonhomogeneous boundary value problem:

$$
\left\{\begin{array}{l}
u^{\prime}-i \Delta u+\varphi(u)=0 \text { in } \hat{Q} \\
u=v \text { on } \hat{\Sigma} \\
u(0)=u^{0} \text { in } \Omega_{0}
\end{array}\right.
$$

where $i^{2}=-1$. Here and in the sequel the prime notation stands for the time derivative $\partial / \partial t$.

The genuine Schrödinger equation $u^{\prime}-i \Delta u=0$ in the cylindrical case was studied by many authors and among which we can mention C. Fabre [1], E. Machtyngier and G. Lebeau. The mathematical aspects of the noncylindrical case has been analyzed in Miranda and Medeiros [5] and in Bernardi, Bonfanti and Lutteroti. In this article we generalize the diffeomorphism $\tau_{t}: \Omega \rightarrow \Omega_{t}$, given by $\tau_{t}(y)=k(t) y+h(t)$, where $\mathrm{k}$ is a real function defined on the nonnegative real numbers, which is considered in [4].

Several approaches are known to solving the problem of exact boundary controllability. We use Hilbert Uniqueness Method (HUM), idealized by J. L. Lions [3]. Our approach, such as in [1], discuss strong and week solutions and solutions defined by transposition, which are also referred to as ultra weak solutions.

In order to solve the problem (1.1), we turned it into an equivalent problem defined in the cylindrical domain $Q=\Omega \times] 0, T[$ by using the change of variables $u(x, t)=w(y, t)$, where $y=K(t)^{-1}(x), y \in \Omega$ and $x \in \Omega_{t}$. Hence, we obtain

$$
\begin{aligned}
& u^{\prime}(x, t)=w^{\prime}(y, t)-\left\{K(t)^{-1}\left(K^{\prime}(t) y\right)\right\} \cdot \nabla w(y, t), \\
& \Delta u(x, t)=\Delta w(y, t)
\end{aligned}
$$

where $\Delta$ represent the Laplace operator in $x$ or in $y$. Since we have made these considerations, it was obtained, in the cylinder $Q$, the following problem: 


$$
\left\{\begin{array}{l}
w^{\prime}-i \Delta w+\vec{b} \cdot \nabla w+\varphi(w)=0 \text { in } Q \\
w=v \text { on } \Sigma \\
w(0)=w^{0} \text { in } \Omega
\end{array}\right.
$$

where $\vec{b}(y, t)=-K(t)^{-1}\left(K^{\prime}(t) y\right)$. In a explicit way, we take

$$
b_{j}(y, t)=-\sum_{i=1}^{n}\left(\sum_{k=1}^{n} a_{k_{j}}(t) \cdot a_{k i}^{\prime}(t)\right) y_{i} .
$$

We will show the existence of solutions for (1.2) and then we will prove that with these solutions we can to obtain the solutions for (1.1).

For obtain the approximate controllability to problem (1.1) we defined the function g by

$$
g(s)=\left\{\begin{array}{l}
\frac{\varphi(s)-\varphi(0)}{s}, \text { if } s \neq 0, \\
\varphi^{\prime}(0), \text { if } s=0
\end{array}\right.
$$

$\mathrm{g}$ is continuous, because the globally Lipschitz function, belongs to $L^{\infty}(\mathfrak{R})$. We have the problem

$$
\mid \begin{array}{lc}
p^{\prime}-i \Delta p+\vec{b} \cdot \nabla p+\nabla \vec{b} \cdot p+g(y) p=0 & \text { in } Q \\
p=0 \text { on } \Sigma & \left(i^{2}=-1\right) \\
p(x, 0)=p_{0}(x) \text { in } \Omega, &
\end{array}
$$

For the existence, uniqueness and regularity of (1.3) $\mathrm{cf}$. [1].

The knowledge on the weak solution of the homogeneous boundary value problem for the formal adjoint defined from $L w=w^{\prime}-i \Delta w+\vec{b} \cdot \nabla w+\varphi(w)$, has a key role. From this formal adjoint we analyze two different classes to problem

$$
\left\{\begin{array}{l}
w^{\prime}-i \Delta w+\vec{b} \cdot \nabla w+\nabla \vec{b} \cdot w+\varphi(w)=f \text { in } Q, \\
w=v \text { on } \Sigma \\
w(0)=w^{0} \text { in } \Omega,
\end{array}\right.
$$

where $\nabla \vec{b}=\sum_{j=1}^{n} \frac{\partial b_{j}}{\partial y_{j}}$.

We state the hypotheses on $K(t)$ as follows:

$K(t)$ orthogonal matrix continuously differentiable for each $t$ in $[0,+\infty[$.

We denote by $v=\left(v_{x}, v_{t}\right)$ the unity exterior normal vector to $\hat{\Sigma}$. We represent by $v *$ the vector $\frac{v_{x}}{\left|v_{x}\right|}$. In order to facilitate the writing, we represent $y_{j} \frac{\partial w}{\partial y_{j}}$ by $y \cdot \nabla w$. We will also use $(.,$.$) to indicate (f, g)=\int_{\Omega} f \cdot \bar{g} d y$ and $|\cdot|,((\cdot)$,$) and \|\cdot\|$ to represent the inner product and norm, respectively, in $L^{2}(\Omega)$ and $H_{0}^{1}(\Omega)$. 
The rest of the article is organized as follows. Section 2 is devoted to the analysis of the weak solution of associated homogeneous problem to the problem (1.2). In Section 3 we establish a result on the existence and uniqueness of strong solution for the problem that was considered in the Section 2. Section 4 is devoted to the ultra weak solution. In Section 5 we obtain the result on approximate controllability.

\section{EXISTENCE AND UNIQUENESS OF SOLUTIONS TO PROBLEM (1.3)}

\subsection{Weak Solutions}

We say that $w$ is a weak solution of (1.4) if given

$$
w^{0} \in H_{0}^{1}(\Omega) \text { and } f \in L^{1}\left(0, T ; H_{0}^{1}(\Omega)\right)
$$

we obtain

$$
w \in L^{\infty}\left(0, T ; H_{0}^{1}(\Omega)\right), w^{\prime} \in L^{\infty}\left(0, T ; H^{-1}(\Omega)\right), w(0)=w^{0} \text { in } \Omega
$$

and

$$
\int_{0}^{T}\left[-\left(w, \psi^{\prime}\right)+i((w, \psi))+(\vec{b} \cdot \nabla w, \psi)+(\nabla \vec{b} \cdot w, \psi)+(\varphi(w), \psi] d t=\int_{0}^{T}(f, \psi) d t\right.
$$

for all $\psi \in L^{2}\left(0, T ; H_{0}^{1}(\Omega)\right)$ such that $\psi^{\prime} \in L^{2}\left(0, T ; L^{2}(\Omega)\right)$ and $\psi(0)=\psi(T)=0$.

Next, we state our result for problem (1.4).

Theorem 2.1 Suppose that $w^{0}$ and $f$ satisfy the condition (2.1). Then there exists a unique function $w: Q \rightarrow \mathbb{C}$ which is a weak solution of problem (1.4).

Proof. We shall prove of this by Galerkin method. For this end, let $\left(\theta_{j}\right)_{j \in \mathbb{N}}$ be the sequence of solutions of the eigenvalue problem:

$$
\left(\left(\theta_{j}, v\right)\right)=\lambda_{j}\left(\theta_{j}, v\right), j \in \mathbb{N}, v \in H_{0}^{1}(\Omega)
$$

From this we conclude that $\theta_{j} \in H_{0}^{1}(\Omega) \cap H^{m}(\Omega)$. Let $V_{m}$ be the subspace of $H_{0}^{1}(\Omega)$ spanned by $\left[\theta_{1}, \theta_{2}, \ldots, \theta_{m}\right]$ by the $m$ first vectors $\theta_{j}$ and let

$$
w_{m}(t)=\sum_{j=1}^{m} g_{j m}(t) \theta_{j}(y)
$$

be the solution to the Cauchy problem 


$$
\left\{\begin{array}{l}
\left(w_{m}^{\prime}(t), \theta\right)+i\left(\left(w_{m}(t), \theta\right)\right)+\left(\vec{b} \cdot \nabla w_{m}(t), \theta\right)+\left(\nabla \vec{b} w_{m}(t), \theta\right)=(f(t), \theta) \\
\text { for all } \theta \in V_{m} \\
w_{m}(0)=w_{m}^{0} \rightarrow w^{0} \text { in } H_{0}^{1}(\Omega) \text { as } m \rightarrow \infty
\end{array}\right.
$$

By standard methods in differential equations, we can prove the existence of a solution for system (2.4) on some interval $\left[0, t_{m}[\right.$ which can be extended, by the first a priori estimate below, over the interval $[0, T]$, for any real number $T>0$. We need two a priori estimates.

Estimate I. We multiply both sides of the approximate equation in (2.4) by $\overline{g_{j m}(t)}$, conjugate of the complex number $g_{j m}(t)$, adding from $j=1$ until $j=m$, we obtain

$$
\begin{aligned}
& \left(w_{m}^{\prime}(t), w_{m}(t)\right)+i\left(\left(w_{m}(t), w_{m}(t)\right)\right)+\left(\vec{b} \cdot \nabla w_{m}(t), w_{m}(t)\right)+ \\
& +\left(\nabla \vec{b} w_{m}(t), w_{m}(t)\right)=\left(f(t), w_{m}(t)\right) .
\end{aligned}
$$

We consider the real parts of the both sides of the last equality. This yields:

$$
\begin{aligned}
& 2 \operatorname{Re}\left(w_{m}^{\prime}(t), w_{m}(t)\right)=\frac{d}{d t}\left|w_{m}(t)\right|^{2} \\
& 2 \operatorname{Re}\left(\vec{b} \cdot \nabla w_{m}(t), w_{m}(t)\right)=-\left(\nabla \vec{b} w_{m}(t), w_{m}(t)\right)
\end{aligned}
$$

Then, we have

$$
\frac{d}{d t}\left|w_{m}(t)\right|^{2}-\left(\nabla \vec{b} \cdot w_{m}(t), w_{m}(t)+2 \operatorname{Re}\left(\nabla \vec{b} w_{m}(t), w_{m}(t)\right)=2 \operatorname{Re}\left(f(t), w_{m}(t)\right)\right.
$$

Thus

$$
\frac{d}{d t}\left|w_{m}(t)\right|^{2} \leq 3|\nabla \vec{b}|\left|w_{m}(t)\right|^{2}+2|f(t)|\left|w_{m}(t)\right|
$$

Integrating (2.6) over $[0, t], 0 \leq t \leq t_{m}$ and employing Gronwall-Belman inequality, we deduce

$$
\left|w_{m}(t)\right| \leq\left|w_{m}^{0}\right|+\int_{0}^{T}|f(s)| d s+\int_{0}^{t} 3|\nabla \vec{b}|\left|w_{m}(s)\right| d s
$$

Therefore

$$
\left|w_{m}(t)\right| \leq C\left[\left|w^{0}\right|+\int_{0}^{T}|f(s)| d s\right]
$$

where $C=\exp \left(\int_{0}^{t} 3|\nabla \vec{b}| d s\right)=1$. 
Estimate II. In order to obtain estimate for $w_{m}^{\prime}(t)$ we multiply the approximate equation in (2.4) by $\lambda_{j} \overline{g_{j m}(t)}$ and add in $j$. Thus, we obtain

$$
\begin{gathered}
\left(w_{m}^{\prime}(t),-\Delta w_{m}(t)\right)-i\left(\Delta w_{m}(t),-\Delta w_{m}(t)\right)+\left(\vec{b} \cdot \nabla w_{m}(t),-\Delta w_{m}(t)\right)+ \\
+\left(\nabla \vec{b} w_{m}(t),-\Delta w_{m}(t)\right)=\left(f(t),-\Delta w_{m}(t)\right) .
\end{gathered}
$$

From the last equality, considering the real parts of the both sides it holds that

$$
\begin{gathered}
\operatorname{Re}\left[\left(\left(w_{m}^{\prime}(t), w_{m}(t)\right)\right)+\left(\vec{b} \cdot \nabla w_{m}(t),-\Delta w_{m}(t)\right)+\left(\nabla \vec{b} w_{m}(t),-\Delta w_{m}(t)\right)\right]= \\
\operatorname{Re}\left(\left(f(t), w_{m}(t)\right)\right) .
\end{gathered}
$$

By using, Green formula and Gauss lemma, we infer

$$
\begin{aligned}
2 \operatorname{Re}\left(\vec{b} \cdot \nabla w_{m}(t),-\Delta w_{m}(t)\right) & =2 \operatorname{Re}\left(\frac{\partial b_{j}}{\partial y_{j}} \cdot \frac{\partial w_{m}(t)}{\partial y_{j}}, \frac{\partial w_{m}(t)}{\partial y_{j}}\right)- \\
& -2\left(\frac{\partial b_{j}}{\partial y_{j}} \frac{\partial w_{m}(t)}{\partial y_{\ell}}, \frac{\partial w_{m}(t)}{\partial y_{\ell}}\right)+\int_{\Gamma} b_{j} v_{j}\left|\frac{\partial w_{m}(t)}{\partial v}\right|^{2} d \Gamma
\end{aligned}
$$

Because $\nabla \vec{b}$ independent of $t$

$$
2 \operatorname{Re}\left(\nabla \vec{b} \cdot w_{m}(t),-\Delta w_{m}(t)\right)=2\left(\frac{\partial b_{j}}{\partial y_{j}} \cdot \frac{\partial w_{m}(t)}{\partial y_{\ell}}, \frac{\partial w_{m}(t)}{\partial y_{\ell}}\right)
$$

From (2.8), (2.9), (2.10) and (2.11), we deduce

$$
\begin{aligned}
& \frac{d}{d t}\left\|w_{m}(t)\right\|^{2}+2 \operatorname{Re}\left(\frac{\partial b_{j}}{\partial y_{j}} \cdot \frac{\partial w_{m}(t)}{\partial y_{\ell}}, \frac{\partial w_{m}(t)}{\partial y_{\ell}}\right)+ \\
& +\int_{\Gamma} \vec{b} \cdot v\left|\frac{\partial w_{m}(t)}{\partial v}\right|^{2} d \Gamma=2 \operatorname{Re}\left(\left(f(t), w_{m}(t)\right)\right) .
\end{aligned}
$$

Now, in order to obtain and identity for surface integral $\int_{\Gamma} \vec{b} \cdot v\left|\frac{\partial w_{m}(t)}{\partial v}\right|^{2} d \Gamma$ we make use of orthogonal projection from $L^{2}(\Omega)$ into $V_{m}$, which is denoted by $P_{m}$. We observe that if $w \in L^{2}(\Omega)$ then $P_{m} w=\sum_{j=1}^{m}\left(w, \theta_{j}\right) \theta_{j}$.

Multiplying both sides of the approximate equation (2.4) by $\theta_{j}$ and adding for $1 \leq j \leq m$, we infer

$$
w_{m}^{\prime}-i \Delta w_{m}+P_{m}\left(\vec{b} \cdot \nabla w_{m}\right)+\nabla \vec{b} w_{m}=P_{m} f
$$


From the last equality, taking the inner product of both sides with $\vec{b} \cdot \nabla w_{m}$ and integrating over $0 \leq t \leq T$ it holds that

$$
\begin{aligned}
& \operatorname{Im} \int_{0}^{T}\left(w_{m}^{\prime}, \vec{b} \cdot \nabla w_{m}\right) d t-\operatorname{Re} \int_{0}^{T}\left(\Delta w_{m}, \vec{b} \cdot \nabla w_{m}\right) d t+ \\
& +\operatorname{Im} \int_{0}^{T}\left(\vec{b} w_{m}, \vec{b} \cdot \nabla w_{m}\right) d t=\operatorname{Im} \int_{0}^{T}\left(P_{m} f, \vec{b} \cdot \nabla w_{m}\right) d t .
\end{aligned}
$$

We have:

$$
\begin{gathered}
\int_{0}^{T}\left(w_{m}^{\prime}, \vec{b} \cdot \nabla w_{m}\right) d t=\left.\left(w_{m}, \vec{b} \cdot \nabla w_{m}\right)\right|_{0} ^{T}-\int_{0}^{T}\left(w_{m}, \vec{b}^{\prime} \cdot \nabla w_{m}\right) d t- \\
-\int_{0}^{T}\left(w_{m}, \vec{b} \cdot \nabla w_{m}^{\prime}\right) d t
\end{gathered}
$$

and from Gauss lemma it holds that

$$
-\int_{0}^{T}\left(w_{m}, \vec{b}^{\prime} \cdot \nabla w_{m}\right) d t=\int_{0}^{T}\left(\vec{b}^{\prime} w_{m}, w_{m}\right) d t+\int_{0}^{T}\left(\vec{b}^{\prime} \cdot \nabla w_{m}, w_{m}\right) d t
$$

and

$$
-\int_{0}^{T}\left(w_{m}, \vec{b} \cdot \nabla w_{m}^{\prime}\right) d t=\int_{0}^{T}\left(\vec{b} w_{m}, w_{m}^{\prime}\right) d t+\int_{0}^{T}\left(\vec{b} \cdot \nabla w_{m}, w_{m}^{\prime}\right) d t
$$

Remark 2.1 In the proof of Theorem 2.1 we utilize the following identities:

$$
\begin{aligned}
\int_{\Gamma} \vec{b} \cdot v\left|\frac{\partial w_{m}(t)}{\partial v}\right|^{2} d \Gamma & =\frac{d}{d t} \operatorname{Im}\left(w_{m}(t), \vec{b} \cdot \nabla w_{m}(t)\right)+\operatorname{Im}\left(\vec{b}^{\prime} \cdot \nabla w_{m}(t), w_{m}(t)\right)+ \\
& +\operatorname{Im}\left(\vec{b} \cdot \nabla w_{m}(t), \nabla \vec{b} \cdot w_{m}(t)\right)-\operatorname{Im}\left(f(t), \nabla \vec{b}^{\prime} \cdot w_{m}(t)\right)+ \\
& 2 \operatorname{Re}\left(\frac{\partial w_{m}(t)}{\partial y_{\ell}}, \frac{\partial b_{j}(t)}{\partial y_{\ell}} \cdot \frac{\partial w_{m}(t)}{\partial y_{j}}\right)+2 \operatorname{Im}\left(\nabla \vec{b} \cdot w_{m}(t), \vec{b} \cdot \nabla w_{m}(t)\right)- \\
& -2 \operatorname{Im}\left(P_{m}(f), \vec{b} \cdot \nabla w_{m}(t)\right) .
\end{aligned}
$$

We consider $P_{m}$ the orthogonal projection from $L^{2}(\Omega)$ in $V_{m}$.

\section{STRONG SOLUTIONS}

The next theorem establish the existence and uniqueness for more regular solution. So, we consider

$$
w_{0} \in H_{0}^{1}(\Omega) \cap H^{2}(\Omega), \quad f \in L^{1}\left(0, T ; H_{0}^{1}(\Omega)\right), \quad f^{\prime} \in L^{1}\left(0, T ; L^{2}(\Omega)\right) .
$$

The solution obtained from of above asumptions is called strong solution of the boundary value problem (2.2).

Theorem 3.1 Suppose that $w^{0}$ and $f$ satisfy the condition (3.1). Then there exists a unique function $w: Q \rightarrow \mathbb{C}$ strong solution of problem (2.2) satisfying 


$$
\begin{gathered}
w \in C^{0}\left([0, T] ; H_{0}^{1}(\Omega)\right), \\
w^{\prime}-i \Delta w+\vec{b} \cdot \nabla w+\nabla \vec{b} \cdot w+\varphi(w)=f \quad \text { a.e. in } Q, \\
w(0)=w^{0} \text { in } \Omega .
\end{gathered}
$$

\section{ULTRA WEAK SOLUTIONS OR SOLUTIONS BY TRANSPOSITIONS}

In this section we consider the non homogeneous mixed problem

$$
\left\{\begin{array}{l}
w^{\prime}-i \Delta w+\vec{b} \cdot \nabla w+\varphi(w)=0 \text { in } Q \\
w=v \text { on } \Sigma \\
w(0)=w^{0} \text { in } \Omega
\end{array}\right.
$$

We suppose $v \in L^{2}(\Sigma)$ and $w^{0} \in H^{-1}(\Omega)$. From an heuristic method, we can give a concept of solutions for (4.1).

Definition 4.1 For $w^{0} \in H^{-1}(\Omega)$ and $v \in L^{2}(\Sigma)$, we define the solution by transposition or ultra weak solution of the nonhomogeneous boundary value problem (4.1), the unique function $w \in L^{\infty}\left(0, T ; H^{-1}(\Omega)\right)$ such that

$$
\int_{0}^{T}\langle w(t), f(t)\rangle_{H^{-1}(\Omega) \times H_{0}^{1}(\Omega)} d t=\left\langle w^{0}, \theta(0)\right\rangle-i \int_{\Sigma} v \frac{\partial \bar{\theta}}{\partial v} d \Gamma d t
$$

for all $f \in L^{1}\left(0, T ; H_{0}^{1}(\Omega)\right)$ and $\theta$ is the weak solution of the back ward transpose problem

$$
\left\{\begin{array}{l}
\theta^{\prime}-i \Delta \theta+\vec{b} \cdot \nabla \theta+\nabla \vec{b} \cdot \theta+g(\theta)=f \text { in } Q \\
\theta=0 \text { on } \Sigma \\
\theta(T)=0 \text { in } \Omega
\end{array}\right.
$$

Remark. Given $\psi_{0} \in L^{2}(\Omega)$ and $h \in L^{2}\left(0, T ; L^{2}(\Omega)\right)$, there exists only one function $\psi: Q \rightarrow \mathbb{C}$, in the sense of distributions on $Q$, satisfying:

$$
\psi \in C\left([0, T] ; L^{2}(\Omega)\right)
$$

such that

$$
-\int_{Q} \psi \overline{\left[\phi^{\prime}-i \Delta \phi+\vec{b} \cdot \nabla \phi+g(\phi)\right]} d x d t=\int_{Q} h \cdot \bar{\phi} d x d t \text {, for all } \phi \in \mathcal{D}(Q)
$$




\subsection{Optimal System}

Theorem 4.1 Given $z_{0} \in L^{2}(\Omega)$, the optimal system associated a (4.1) for $y_{0}=0$ is:

$$
\begin{aligned}
& \mid \begin{array}{l}
y^{\prime}-i \Delta y+\varphi(y)+\vec{b} \cdot \nabla y+k p \cdot \chi_{w}=0 \text { in } Q \\
y=0 \text { on } \Sigma \\
y(0)=0 \text { in } \Omega .
\end{array} \\
& \mid \begin{array}{l}
-p^{\prime}-i \Delta p+\vec{b} \cdot \nabla p+g(p)=0 \text { in } Q \\
p=0 \text { on } \Sigma \\
p(T)=y(T)-z_{0} \text { in } \Omega .
\end{array}
\end{aligned}
$$

Proof:

We consider the problem of following controls:

If $y=y(v)$ is the solution of (4.1), for each $k \in N$ let us consider the functional

$$
J_{k}(v)=\frac{1}{2}|\nu|_{L^{2}(w \times(0, T))}^{2}+\frac{k}{2}\left|y(T, v)-z_{0}\right|_{L^{2}(\Omega)}^{2}, \text { where } k>0
$$

and minimization problem

$$
\left(P_{k}\right) \mid \begin{aligned}
& \operatorname{Min} J_{k}(v) \\
& \text { for all } v \in L^{2}(w \times(0, T))
\end{aligned}
$$

Then there exists a solution $v_{k}$ of $\left(P_{k}\right)$, for each $k \in N$. The derivative of $J_{k}(v)$ is given by:

$$
J_{k}^{\prime}(v) \cdot \xi=\int_{w \times(0, T)} v \cdot \bar{\xi} d x d t+k\left(y(v, T)-z_{0}, y(\xi, T)\right)
$$

for all $\xi \in L^{2}(w \times(0, T))$.

If we evaluate $J_{k}^{\prime}(v) \cdot \xi$ in the solution $v_{k}$ of $\left(P_{k}\right)$ we must have the Euler equation

$$
J_{k}^{\prime}\left(v_{k}\right) \cdot \xi=\int_{w \times(0, T)} v_{k} \cdot \bar{\xi} d x d t+k\left(y_{k}\left(v_{k}, T\right)-z_{0}, y(\xi, T)\right)
$$

for all $\xi \in L^{2}(w \times(0, T))$.

We denoted by $y_{k}=y\left(v_{k}\right)$ solution of system

$$
\mid \begin{aligned}
& y_{k}^{\prime}-i \Delta y_{k}+\varphi\left(y_{k}\right)+\vec{b} \cdot \nabla y_{k}=v_{k} \cdot \chi_{w} \text { in } Q \\
& y_{k}=0 \text { on } \Sigma \\
& y_{k}(x, 0)=y_{0}(x) \text { in } \Omega,
\end{aligned}
$$

and we introduced $p_{k}$ solution of system 


$$
\mid \begin{aligned}
& p_{k}^{\prime}-i \Delta p_{k}+\vec{b} \cdot \nabla p_{k}+g\left(p_{k}\right)=0 \text { in } Q \\
& p_{k}=0 \text { on } \Sigma \\
& p_{k}(x, T)=y_{k}(T)-z_{0} \text { in } \Omega,
\end{aligned}
$$

By (4.8) and (4.9) we obtain

$$
-\left(y_{k}(T)-z_{0}, y(\xi, T)\right)+\int_{\omega \times(0, T)} p_{k} \cdot \bar{\xi} \cdot \chi_{\omega} d x d t=0
$$

By the Euler equation

$$
\int_{\omega \times(0, T)}\left(v_{k}+k \cdot p_{k}\right) \cdot \bar{\xi} \cdot \chi_{\omega} d x d t=0, \text { for all } \xi
$$

Then

$$
v_{k}+k \cdot p_{k} \cdot \chi_{\omega}=0
$$

We obtain the optimal system by passaged the limit in $\mathrm{k}$.

\subsection{Approximate controllability}

Theorem 4.2 Given $y_{0} \in L^{2}(\Omega), \varphi(0)=0$ and $v \in L^{2}(w \times(0, T))$ the problem (??) have approximate controllability in $L^{2}(\Omega)$.

\section{Proof:}

Let $f \in L^{2}(\Omega)$ such has $(y(T), f)=0, \forall v \in L^{2}(w,(0, T))$

We defined $\psi=\psi(x, t)$ solution the

$$
\mid \begin{aligned}
& \psi^{\prime}-i \Delta \psi=0 \text { in } Q \\
& \psi=0 \text { on } \Sigma \\
& \psi(x, T)=f(x) \text { in } \Omega,
\end{aligned}
$$

Multiplying (4.10) by $y=y(x, t)$ we obtained

$$
-(y(T), f)+(\psi, v)=0, \forall v \in L^{2}(w \times(0, T))
$$

Then

$$
(\psi, v)=0, \forall v \in L^{2}(w \times(0, T)) \Rightarrow \psi=0 \text { in } w \times(0, T)
$$

By Mizohata' theorem $\psi=0$ in $\Omega \times(0, T)$ therefore $f=0$

Let $z_{0} \in L^{2}(\Omega), \varepsilon>0$, exist $v_{0} \in L^{2}(w \times(0, T))$ such that 


$$
\left|y\left(t, v_{0}\right)-z_{0}\right|<\varepsilon
$$

We have $J_{k}\left(v_{k}\right) \leq J_{k}\left(v_{0}\right) \Rightarrow k\left|y_{k}(T)-z_{0}\right|^{2} \leq\left|v_{0}\right|_{L^{2}(w \times(0, T))}+k\left|y\left(T, v_{0}\right)-z_{0}\right|^{2}$ what implies

$$
\left|y_{k}(T)-z_{0}\right|^{2} \leq \frac{\left|v_{0}\right|_{L^{2}(w \times(0, T))}^{2}}{k}+\left|y\left(T, v_{0}\right)-z_{0}\right|^{2}
$$

Then we have construct the approximate sequence in $E(T)$ for all $z_{0} \in L^{2}(\Omega)$.

\section{REFERENCIAS BIBLIOGRÁFICAS}

[1] FABRE, C., PAUL, J. P. \& ZUAZUA, E., Approximate controllability of the semilinear heat equation, Proc. Royal Soc., Edinburgh, 125A 31 - 61, (1995).

[2] FERNÁNDEZ, L. A. \& ZUAZUA, E., Approximate controllability for the semilinear heat involving gradient terms, prepinter (to appear on J. Optim, Theory Applic.). (1997).

[3] LIONS, J. L., Remarques sur la contrôlabilité approcheé. Jornadas Hispano-Francesa sobre control de Sistemas Distribuidos, pág. 77 - 87, Universidad de Málaga, España, (1991).

[4] LIMACO, J. \& MEDEIROS, L. A., Approximate controllability in noncylindrical domains, Communications in Applied Analysis, 6, No 3, 375 - 392, (2002).

[5] Milla MIRANDA, M. \& MEDEIROS, L. A., Exact Controllability for Schrödinger equations in non cylindrical domains, Minicurso 41 SBA. 